\title{
A conversation with Robert Schrier
}

A prolific expert in the renal field, Dr. Robert Schrier is professor of medicine at the University of Colorado Denver School of Medicine, where he was chairman of the Department of Medicine for 26 years. Schrier (Figure 1) is an expert in patient-oriented research in acute kidney injury, autosomal dominant polycystic kidney disease, hypertension, and diabetic nephropathy; he is behind most of what we know about fluid balance in the body.

He's written over a thousand scientific articles and several books and has been constantly funded by the NIH for over 45 years. Find more stories, including his recollection of being inducted into the Indiana Basketball Hall of Fame on the JCI website (https://www.jci.org/ videos/cgms).

JCI: Where were you brought up?

Schrier: I'm a Hoosier; it means someone born in Indiana. I played sports. I have an older brother who was always saying, "Let's go play ball!" During the baseball season, we played baseball, and after that was over, we'd go play basketball. And during the football season, we would play football; after that day was over, we would go play basketball that evening.

I was a pretty good student, but I'm embarrassed to say that I never studied. I can never remember studying at home. But I was about fifth out of 300 students in my high school, so I did fine. I did my studying during the study halls; I really just didn't study at home.

When I went off to college, I was deciding where I could go to play basketball, not to study medicine. I visited the campus at Michigan State and then Indiana University and then DePauw University. DePauw offered an academic scholarship, so if I didn't play basketball, I still had a scholarship. When they asked me what my major would be, I wasn't quite sure. My mother was a nurse, and she said, "Just tell them pre-med." So I told them pre-med. That's how my trajectory got started.

JCI: Did you have any sort of academic enlightenment in college?
Schrier: College was different. I did learn to study, to focus.

JCI: How did you balance academic rigor while you were also a college athlete?

Schrier: I played basketball and baseball, and so athletics for me was pretty much year round. I think when you have more to do, you organize yourself better. In life we multitask. It's not very often that we have the privilege of just doing one thing.

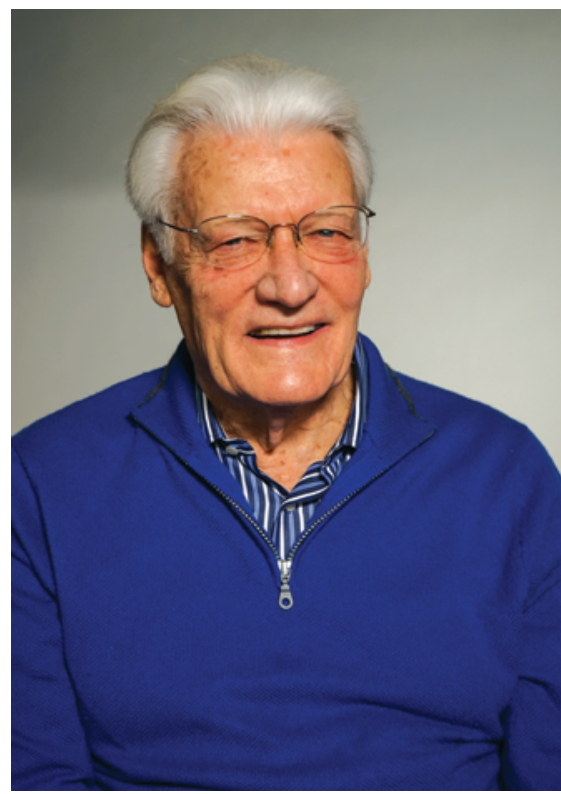

Figure 1. Robert Schrier on April 25, 2015.

Image credit: Karen Guth.

I knew that in medicine there would be a need to know about science and I remember taking chemistry for the first time in college and thinking I should have taken chemistry in high school! I should also have taken physics too... but I survived.

I had a professor in my senior year who said, "Maybe you should take a year off before you go to medical school if you're not absolutely sure what you want to do." I applied for a Fulbright scholarship, which I was fortunate enough to receive. I went to Gutenberg University in Mainz, Germany, and studied anthropology for a year. Professor Von Eichstedt, who I stud- ied with, was great. He was quite famous as an anthropologist, and he said, "What you really want to do is learn about different cultures. Europe has many different cultures, so you should travel quite a bit." I decided that was a great idea, so during my Fulbright, I traveled through the Middle East, through Europe, through Scandinavia. I got to know many different people, and I learned about the diversity of cultures, the diversity of opinions, the diversity of biases and prejudices. I also met my wife, so it was a good period for me.

I came back from Germany, and that's when I went to medical school. I went to Indiana University in Indianapolis. I remember thinking medical school was easier than college, because in college I had a full agenda for practice in basketball and additional travel to different schools for games. In medical school, I played amateur basketball, but I didn't have to travel and practice every day. I was just more focused on the academics.

JCI: Did you ever consider a career in professional athletics?

Schrier: I did. When I was a senior in high school playing on a summer baseball team, a scout came to see me; he had signed Gil Hodges and Carl Erskine to the Brooklyn Dodgers. They became very famous. And they also had Jackie Robinson, Pee Wee Reese, Carl Furillo, and Roy Campanella. I was invited to go to their spring training camp. But I learned that if you played professional sports, in any sport, you couldn't play any college sports. I wanted to play college basketball, so I thought, if they want me now, maybe after I finish college, they might still want me then. It didn't happen, but that would have been fun, though, to be down there with those guys.

JCI: After you finished medical school, what was your trajectory?

Schrier: I took my internship in Marion County Hospital. I had gotten married by then, and we thought it would be good to look someplace beyond Indiana for our next home. One of my professors from Indiana University had mentioned that there was a great program in Seattle, at the University of Washington. He knew one of the professors there, and he thought he might 
be able to get me a position. I did transfer to the University of Washington and finished my internal medicine residency there.

JCI: When during this process did you start doing research?

Schrier: I hadn't done any research in medical school, and when I was at Harvard for a fellowship in endocrine metabolism, I received a really nice invitation from Uncle Sam during the Vietnam War to join the armed forces, specifically the United States Army Medical Corps. They gave me two alternatives. I could be drafted for two years, and I think there was something mentioned about Columbia, South Carolina, but this option probably also included a tour in Vietnam. Or I could go for three years to Walter Reed Army Medical Center and their Institute of Research. I thought I was probably headed for academic medicine, where I could see patients and teach and do research, so I chose the three years at Walter Reed, and it was a great decision. That era is when I started to become involved in research.

During basic training, many recruits were not acclimatized to the heat and humidity, especially those who were not in good physical shape. They were brought to San Antonio, and some of the exercises were quite rigorous and several recruits ended up with heat stroke. The colonel at Walter Reed Medical Center told us, "If you have heat stroke and you have kidney failure, you have high mortality." I asked why, and he said that high temperature burns up all the organs - the brain, liver, and kidneys.

Somehow that didn't seem right to me; we went back and looked up five fatal cases. When you develop kidney failure, you accumulate uremic toxins, and one thing we could measure as a surrogate was blood and urine hydrogen $[\mathrm{pH}]$. The patients were getting peritoneal dialysis, where the fluid is put in the abdomen, poisons come out, and you throw the contaminated fluid away.

And despite the continuous peritoneal dialysis, blood and urine hydrogen just kept rising. I remember talking to the colonel, who was considered an expert in peritoneal dialysis (in contrast to hemodialysis, where you take the blood out, send it through a kidney, put the "cleaned" blood back in). I argued that the patients who died were underdialyzed. The implication was that had they been better dialyzed, there might not be an $80 \%$ mortality with heat stroke and kidney failure.

He was unwavering and said, "Well, let's start out with peritoneal dialysis," because that was his thing, "and if it doesn't seem to control the uremic toxins, then we'll switch to hemodialysis." We probably did peritoneal dialysis for an hour and then got these boys on hemodialysis. That summer, we had 10 patients and we had an $80 \%$ survival.

After that experience, I said, "Research is important."

JCI: This honed your focus to renal research?

Schrier: After I finished my time in the armed forces, I was looking around for a job at a university. I visited a few places and decided to go to the University of California San Francisco, which was a very wellknown medical center; I was able to work under the inimitable Holly Smith. I was there for three years and would have stayed longer, but the University of Colorado called, in need of a chief of their Kidney Division. I've been in Colorado ever since, 20 years as head of Kidney. After four years, the Chairman of Medicine stepped down and they did a national search. I was not looking to fill that job; I was happy with the Kidney Division, but they eventually ended up asking me to take the chairmanship. I had 16 years where I overlapped doing head of the Kidney Division and Chairman of the entire Department of Medicine. It was challenging, but it was great fun.

JCI: What are you most proud of accomplishing there?

Schrier: In those 26 years, we went from about $\$ 3$ million in annual clinical earnings to about $\$ 300$ million. In research, we went from $\$ 3$ million research funding to about $\$ 100$ million. We went from 75 faculty to 500 faculty and became nationally prominent.

JCI: Do you think that your approach to being a leader and a chairman was shaped at all by your experience being coached in athletics?

Schrier: I think it is similar. You try to lead by example. You try to use positive reinforcement. If you do those two things, you don't need much negative reinforcement.

JCI: You've also written a voluminous amount; your CV is one of the thickest I've ever received. You've penned over a thousand articles and several textbooks in many different editions as well as a few books. Has writing always come easily to you?

Schrier: I enjoy writing. When you've done something for 40 or 50 years, the numbers add up, so it's not as much as it might seem. Only two-thirds of them were original studies; the rest were reviews, textbook chapters, and so forth.

And I worked on common things. Polycystic kidney disease is the fourth most common cause of end-stage kidney disease, and one out of every 500 to 1,000 people has polycystic kidney disease. It's autosomal dominant, so if you have it, you have a chance that $50 \%$ of your offspring are going to have polycystic kidney disease as well.

I also worked on a hormone called vasopressin, and in that realm, my timing was good. People thought we knew all there was to know about water regulation, but I didn't agree. The immunoassay for vasopressin came along; we were able to actually measure its levels, and it turns out that arterial underfilling, which gives a bad prognosis, stimulates the nonosmotic release of vasopressin. It's called nonosmotic because osmotically, you don't get hyponatremia. You drink a glass of water, osmolality falls, the antidiuretic hormone is turned off, and the glass of water comes out in the urine. But when you have a nonosmotic stimulus, heart failure, cirrhosis, nephrotic syndrome, or an array of diseases, you get hyponatremia. We defined the arterial baroreceptor release of vasopressin, which can override osmotic release of vasopressin.

JCI: Most of your research is focused on patient-oriented, clinical research. Why never delve into animal models?

Schrier: Basic research is enormously important, and it gives you the underpinnings for understanding clinical research, but sometimes it doesn't correlate. If you can actually do the research in patients, that's the ultimate for a clinical investigator like me.

JCI: What do you think you would do if you were not a physician-scientist? Coach? Writer?

Schrier: Probablyboth. I think the young people that I've trained over the years, after family, have been the most rewarding. So, I'm kind of a coach and mentor.

\section{Ushma S. Neill}

